\title{
Correlates of poor mental health in early pregnancy in obese European women
}

Matteo C. Sattler', Judith G. M. Jelsma ${ }^{1}$, Annick Bogaerts ${ }^{3}$, David Simmons ${ }^{4}$, Gernot Desoye ${ }^{5}$, Rosa Corcoy ${ }^{6,7}$, Juan M. Adelantado ${ }^{6}$, Alexandra Kautzky-Willer ${ }^{8}$, Jürgen Harreiter ${ }^{8}$, Frans A. van Assche ${ }^{9}$, Roland Devlieger ${ }^{9}$, Goele Jans ${ }^{9}$, Sander Galjaard ${ }^{9,10}$, David Hill' ${ }^{11}$, Peter Damm ${ }^{12}$, Elisabeth R. Mathiesen ${ }^{12}$, Ewa Wender-Ozegowska ${ }^{13}$, Agnieszka Zawiejska ${ }^{13}$, Kinga Blumska ${ }^{13}$, Annunziata Lapolla ${ }^{14}$, Maria G. Dalfrà ${ }^{14}$, Alessandra Bertolotto ${ }^{15}$, Fidelma Dunne ${ }^{16}$, Dorte M. Jensen ${ }^{17}$, Lise Lotte T. Andersen ${ }^{17}$, Frank J. Snoek ${ }^{18,19}$ and Mireille N. M. van Poppel ${ }^{12^{*}}$

\begin{abstract}
Background: Depression during pregnancy is associated with higher maternal morbidity and mortality, and subsequent possible adverse effects on the cognitive, emotional and behavioral development of the child. The aim of the study was to identify maternal characteristics associated with poor mental health, in a group of overweight/ obese pregnant women in nine European countries, and thus, to contribute to better recognition and intervention for maternal depression.

Methods: In this cross-sectional observational study, baseline data from early pregnancy ( $<20$ weeks) of the DALI (Vitamin D and Lifestyle Intervention for gestational diabetes mellitus prevention) study were analyzed. Maternal mental health was assessed with the World Health Organization Well-Being Index (WHO-5). Women were classified as having a low $(\mathrm{WHO}-5 \leq 50)$ or high wellbeing.
\end{abstract}

Results.: A total of 735 pregnant women were included. The prevalence of having a low wellbeing was 27.2\%, 95\% Cl [24.0, 30.4]. Multivariate analysis showed independent associations between low wellbeing and European ethnicity, $O R=.44,95 \% \mathrm{Cl}[.25, .77]$, shift work, $O R=1.81,95 \% \mathrm{Cl}[1.11,2.93]$, insufficient sleep, $O R=3.30,95 \% \mathrm{Cl}$ $[1.96,5.55]$, self-efficacy, $O R=.95,95 \% \mathrm{Cl}[.92, .98]$, social support, $O R=.94,95 \% \mathrm{Cl}[.90, .99]$, and pregnancy-related worries (socioeconomic: $O R=1.08,95 \% \mathrm{Cl}[1.02,1.15]$; health: $O R=1.06,95 \% \mathrm{Cl}[1.01,1.11]$; relationship: $O R=1.17$, $95 \%$ Cl $[1.05,1.31])$.

Conclusions: Mental health problems are common in European overweight/obese pregnant women. The identified correlates might help in early recognition and subsequent treatment of poor mental health problems during pregnancy. This is important to reduce the unfavorable effects of poor mental health on pregnancy outcomes.

Trial registration: ISRCTN70595832, 02.12.2011.

Keywords: Mental health, Depression, Pregnancy, Obesity

\footnotetext{
* Correspondence: mireille.van-poppel@uni-graz.at

${ }^{1}$ Institute of Sport Science, University of Graz, Mozartgasse 14, 8010, Graz,

Austria

${ }^{2}$ Department of Public and Occupational Health, Amsterdam Public Health

Research Institute, VU University Medical Centre, Amsterdam, the

Netherlands

Full list of author information is available at the end of the article
} International License (http://creativecommons.org/licenses/by/4.0/), which permits unrestricted use, distribution, and reproduction in any medium, provided you give appropriate credit to the original author(s) and the source, provide a link to the Creative Commons license, and indicate if changes were made. The Creative Commons Public Domain Dedication waiver (http://creativecommons.org/publicdomain/zero/1.0/) applies to the data made available in this article, unless otherwise stated. 


\section{Background}

The statement of the World Health Organization (WHO) that there can be no "health without mental health" [1] emphasizes the importance of mental health. Almost $30 \%$ of all people experience serious mental health problems, such as mood or anxiety disorders, across their lifetime [2]. Depression alone has a cross-cultural lifetime prevalence from 1 to $17 \%$ [3] and has a substantial influence both on disability and mortality [4]. It is associated with various adverse health conditions such as unexplained somatic symptoms [5], cardiovascular disease [6, 7], type 2 diabetes [8], HIV/AIDS [9] and tuberculosis [10]. In addition, depression is associated with obesity (body mass index $(\mathrm{BMI}) \geq 30 \mathrm{~kg} / \mathrm{m}^{2}$ ) [11] and this association becomes stronger at an older age [12].

Moreover, depression is one of the more common complications during pregnancy [13] with a prevalence of up to $11 \%$ [14]. Pregnancy-related maternal depression has been found to be associated with obesity [15], poor socioeconomic status (including occupation, education and income), lack of social support, history of domestic violence or abuse, personal history of mental illness, unplanned pregnancy, adverse life events and high perceived stress, smoking, single status, past or present pregnancy complications and pregnancy loss $[16,17]$. Furthermore, maternal depression appears to be inversely related with physical activity (PA) [18] and there is evidence to suggest that PA can help to reduce antenatal depression [19] as well as improve maternal physical health [20].

Depression in pregnancy not only affects the health of the woman, but also, potentially, the unborn child. The fetal period is a sensitive and challenging period, where changes and harm could have short- and long-term consequences for the development of the offspring. In contrast to parental genetic factors, fetal programming (e.g., programming of the child's stress response system) supposes intra uterine developmental origins of future health and disease, mainly through epigenetic mechanism [21]. Thus, a stressful in utero environment (e.g., poor maternal mental health) could lead to detrimental consequences for the offspring's physical and mental health $[22,23]$.

Indeed, there is substantial evidence that pregnancyrelated depression is associated with higher risks for negative child outcomes, potentially lasting until late adolescence [24]. For instance, depression in pregnancy represents a risk factor for adverse birth outcomes such as preterm birth (< 37 weeks of gestation), low birth weight $(<2500 \mathrm{~g})$, small-for-gestational age $[25,26]$ and poorer behavioral, neurophysiological and cognitive development (e.g., emotional and immune functioning) of the child $[27,28]$. Consequently, there is no other period in life where the statement "there is no health without mental health" is more true than during pregnancy [29].

Since obesity and depression are often comorbid [11], it is important to investigate mental health in obese pregnant women. The increasing prevalence of overweight $\left(B M I \geq 25 \mathrm{~kg} / \mathrm{m}^{2}\right)$ and obese $\left(B M I \geq 30 \mathrm{~kg} / \mathrm{m}^{2}\right)$ pregnant women is by itself already a growing public health concern worldwide [30, 31]. Maternal obesity increases the risk for pregnancy-related complications such as neonatal intensive care requirement, infection and haemorrhage [32] and may even affect future health of the offspring [33]. Although extensive research on the impact of obesity on maternal physical health has been carried out, less attention has been paid to relationship of obesity and maternal mental health. In particular, a better understanding of factors associated with poor mental health in obese pregnant women, and opportunities for early recognition and management is most important.

However, studies concerning mental health in pregnancy were often performed in low-risk populations such as women without serious health conditions and European-wide studies on a broad range of maternal mental health correlates are sadly lacking. This study combines these two aspects with the objective to explore the role of correlates, including lifestyle, socioeconomic, biological, and pregnancy-specific factors, associated with poor mental health in early pregnancy in a large group of overweight/obese European women.

\section{Methods}

\section{Study design and setting}

This cross-sectional observational study was part of the DALI (Vitamin D and Lifestyle Intervention for gestational diabetes mellitus prevention) project (ISRCTN70595832), which aimed to identify the best available preventive intervention (healthy eating (HE), PA, vitamin D) for gestational diabetes mellitus in a European cohort of obese pregnant women. Therefore, following a pilot study [34], a randomized controlled trial was implemented in nine European countries: Austria, Belgium, Denmark, Italy, Ireland, the Netherlands, Poland, Spain and the United Kingdom. Detailed study design and data collection of the DALI project were published previously [35]. Participants were recruited from January 2012 to April 2015 by participating hospitals, obstetrician, midwifes and general practices and randomly allocated to one of the eight intervention arms (HE, PA, HE \& PA, HE \& PA \& vitamin D, HE \& PA \& placebo, vitamin D alone, placebo alone, control). Measurements took place at baseline (< 20 weeks of gestation), 24-28 weeks, 35-37 weeks and after delivery. Participants receiving the lifestyle interventions were assigned a lifestyle coach and those receiving the vitamin $\mathrm{D}$ interventions were asked to take four 
vitamin $\mathrm{D}$ tablets per day (each containing on average 400 IU vitamin D) from randomization until delivery. The lifestyle coaching was based on principles of patient empowerment and cognitive behavioral techniques inspired by motivational interviewing and included five face-to-face sessions, up to four telephone sessions, handbooks and educational materials in order to increase PA, HE or both.

For the purpose of the present study, cross-sectional data from early pregnancy ( $<20$ weeks; baseline) of the pilot study, Lifestyle trial and Vitamin D trial were used. The study received ethical approval in all nine countries. All participants provided written informed consent.

\section{Participants}

Inclusion criteria were defined as singleton pregnancy, less than 20 weeks of gestation, pre-pregnancy $\mathrm{BMI} \geq$ $29 \mathrm{~kg} / \mathrm{m}^{2}$ (based on self-reported weight and measured height) or BMI $\geq 29 \mathrm{~kg} / \mathrm{m}^{2}$ at baseline measurement and age $\geq 18$ years. The BMI cut off was based on data on the European obesity prevalence to ensure sufficiency in the recruiting process for countries with lower rates of maternal obesity [36]. Other exclusion criteria were preexisting diabetes, inability to walk $\geq 100 \mathrm{~m}$ safely, multiple pregnancy, requiring a complex diet, significant chronic medical condition or psychiatric disease, unable to speak the major language of the country of recruitment fluently or unable to converse with the lifestyle coach in another language for which translated materials existed.
Of the 3544 women approached, 1069 (30.2\%) consented to participate. Of those, a total of 738 women between 18.7 and 47.1 years $(M=32.0, S D=5.4)$ fulfilled all inclusion criteria and were randomized and included at baseline. Seventy-six (10.3\%) were overweight at baseline $(\mathrm{BMI}<30)$. Please note that from now on we refer to the whole sample as obese, including this percentage of overweight pregnant women. Three of 738 women did not complete mental health measurements, and therefore, were excluded from the present study. Sociodemographic characteristics are presented in Table 1 in detail. Number of missing data was below $5 \%$ for all variables (see Table 1), except for heart rate data (5.6\%). Differences between missing and non-missing data were assessed if there were more than $5 \%$ missing values for one variable.

\section{Measures}

\section{Mental health}

Assessment of maternal mental health was based on the World Health Organization Well-Being Index (WHO-5), a widely-used, unidimensional instrument measuring subjective wellbeing [37]. It has shown to be a valid screening instrument for clinical depression with high sensitivity and specificity, and has been validated for all languages required in the present multi-national study $[38,39]$.The scale consists of five items, each rated on a 6-point Likert scale (0: at no time, 5: all of the time), and following total scores, standardized scores $(0-100)$ are calculated. Based on current literature on sensitivity and specificity for clinical depression, values $\leq 50$ were

Table 1 Maternal socio-demographic characteristics

\begin{tabular}{|c|c|c|c|}
\hline Characteristic & $\begin{array}{l}\text { Total } \\
(N=735)\end{array}$ & $\begin{array}{l}\text { Low wellbeing }^{\text {a }} \\
(n=200)\end{array}$ & $\begin{array}{l}\text { High wellbeing } \\
(n=535)\end{array}$ \\
\hline Age, years $(M \pm S D)$ & $32.0 \pm 5.4$ & $31.3 \pm 5.9$ & $32.3 \pm 5.2$ \\
\hline $\mathrm{BMI}, \mathrm{kg} / \mathrm{m}^{2}$ (median (IQR)) & $33.4(31.4-36.6)$ & $33.4(31.7-36.9)$ & $33.4(31.3-36.6)$ \\
\hline Pre-pregnancy BMI, kg/m² (median (IQR)) & $32.7(30.5-35.8)$ & $33.0(30.9-35.7)$ & $32.5(30.2-35.8)$ \\
\hline Ethnicity, European descent ( $n(\%))$ & $638(86.8)$ & $160(80.0)$ & $478(89.3)$ \\
\hline \multicolumn{4}{|l|}{ Education ( $n(\%)$, total = 734) } \\
\hline Low (no qualification/intermediate) & $89(12.1)$ & $28(14.0)$ & $61(11.4)$ \\
\hline Medium (higher school/apprenticeship) & $233(31.7)$ & $75(37.5)$ & $158(29.5)$ \\
\hline High (diploma/university) & $412(56.1)$ & $96(48.0)$ & $316(59.1)$ \\
\hline \multicolumn{4}{|l|}{ Occupational status ( $n(\%)$, total = 733) } \\
\hline Home duties & $63(8.6)$ & $21(10.5)$ & $42(7.9)$ \\
\hline Unemployed/not able to work & $109(14.8)$ & $37(18.5)$ & $72(13.5)$ \\
\hline Working (fulltime/part-time/student) & $561(76.3)$ & $141(70.5)$ & $420(78.5)$ \\
\hline Household composition, living with partner (n (\%)) & $672(91.4)$ & $177(88.5)$ & $495(92.5)$ \\
\hline Marital status, with partner $(n(\%)$, total $=734)$ & $690(93.9)$ & $179(89.5)$ & $511(95.5)$ \\
\hline Shift work, yes ( $n(\%)$, total = 730) & $149(20.3)$ & $52(26.0)$ & $97(18.1)$ \\
\hline
\end{tabular}

${ }^{a}$ Based on the World Health Organization Well-Being Index (WHO-5). Values $\leq 50$ were considered as low wellbeing (poor maternal mental health), values $>50$ as high wellbeing 
considered as low wellbeing (poor maternal mental health), values above 50 as high wellbeing [38]. Cronbach Alpha $(\alpha)$ was 0.82 for the whole scale.

\section{Potential correlates}

The selection of variables collected at baseline was based on current literature $[7,16,17]$ and then clustered into 4 groups: a) socio-demographic (age, pre-pregnancy BMI, BMI at baseline, ethnicity, education, household composition, occupational status, shift work, marital status), b) lifestyle (smoking, alcohol consumption, hours of sleep per day $(24 \mathrm{~h})$, days of insufficient sleep per month, snoring, PA), c) biological (polycystic ovary syndrome, first degree relative with diabetes, fasting plasma glucose, fasting plasma insulin, Homeostasis Model Assessment of Insulin Resistance (HOMA-IR), systolic and diastolic blood pressure, chronic hypertension, resting heart rate) and d) pregnancy characteristics (weeks of gestation (at baseline), pregnant before, number of own children, previous stillbirths/miscarriages, previous fetal macrosomia, previous congenital malformation, previous gestational diabetes, pre-pregnancy to baseline weight gain, attitude/importance of current weight, social support, outcome expectancies, self-efficacy, pregnancyrelated worries). Physiological parameters (plasma glucose, plasma insulin, blood pressure, heart rate) were quantified by standardized, commercially available devices, whereas all other variables were self-reported.

Hours of sleep per day (24 h), snoring and insufficient sleep were retrieved from single items (How many hours do you sleep per day on average; How many days in the last month have you had the feeling of insufficient sleep; How many days per week do you snore/are told you snore). Sleep hours per day were categorized into $\leq 6 \mathrm{~h}$, 6-9 h and $\geq 9$ h [40], ethnicity into European and nonEuropean, education into low (no qualification/intermediate), medium (higher school/apprenticeship) and high (diploma/university), occupational status into working, not working and home duties, household composition into living with partner and not living with partner, marital status into with partner and without partner.

PA was based on the pregnancy physical activity questionnaire (PPAQ), which is a valid instrument for the assessment of PA during pregnancy [41]. In addition to 32 original activities, two more questions concerning cycling to work and cycling for fun have been added. Time spent in each activity was asked, and then multiplied by its intensity, resulting in an average weekly energy expenditure (MET hours/week) for each activity. After categorization of activities into sedentary, light, moderate or vigorous, total MET hours/week of moderate-tovigorous physical activity (MVPA) and sedentary behavior $(\mathrm{SB})$ were used for analysis.
Pregnancy-related worries have shown to be related to mood outcomes and were assessed by the Cambridge Worry Scale [42], a 13-item questionnaire (6-point Likert scale; 0: not a worry, 5: major worry) measuring worries across four domains: sociomedical $(\alpha=.71)$, socioeconomic $(\alpha=.59)$, health $(\alpha=.66)$ and relationship $(\alpha=.61)$. Cronbach Alpha for the whole scale was .77. Domain scores were used for analysis. Attitude (e.g., It is important for me to manage my weight), social support (e.g., I am satisfied with the level of support I am receiving for eating healthily from my partner, family and friends), outcome expectancies (e.g., Staying physically active during this pregnancy, will help to reduce health risks for my baby) and self-efficacy (e.g., I am confident that I will succeed in managing my weight) were based on the Health Action Process Approach (HAPA) model [43], referring to PA, nutrition and weight management during pregnancy, and collected with items on a 10point Likert scale. Attitude to current weight as well as social support consisted of two items $\left(\alpha_{\mathrm{a}}=.76, \alpha_{\mathrm{s}}=.87\right)$, outcome expectancies of six items $(\alpha=.93)$ and selfefficacy of five items $(\alpha=.88)$. Sum scores of each dimension were used for further analysis.

\section{Statistical analysis}

Statistical analyses were performed using SPSS Data Analysis version 22.0 (IBM Corp, Armonk, NY, USA). Descriptive statistics for all variables were calculated, including mean and standard deviation for continuous variables with normal distribution, median and interquartile range (IQR) for continuous variables without normal distribution, and frequencies and percentages for categorical variables. Normal distribution was assessed by Shapiro-Wilk, Q-Q-Plots and Histogram. Evaluation of missing and non-missing data was done by $t$ test, Welch test and Chi square or Fisher's exact tests.

To evaluate the association between poor maternal mental health and potential correlates, logistic regression models were used, comprising a three-step procedure. In all analysis, the outcome was defined as the dichotomous variable derived from the WHO-5 index (low wellbeing vs. high wellbeing). Odds ratios (OR) with 95\% confidence intervals $(\mathrm{CI})$ were calculated for each association. In a first step, bivariate logistic regression models between outcome and each potential correlate were calculated. Significant correlates were included in the next step. In this second step, four multivariate logistic regression models were calculated, set up of blocks with related correlates (1) socioeconomic status (2) sleep (3) worries (4) perceptions and attitudes. All correlates of one block were entered at the same time and significant correlates were included in the next step. In this third and final step, remaining correlates were analyzed in one 
multivariate logistic regression model, entering all correlates at the same time.

Significance in step one and two was considered as $p$ $<.10$ to allay beta error. In step 3 as well as in all other analyses, significance was considered as $p<.05$. BMI at baseline (based on measured weight and height) and pre-pregnancy BMI (based on self-reported weight and measured height) were both included in the first step, but if significant, BMI at baseline was used for step two and three. Linearity between outcome and each potential correlate was assessed by the interaction term between the potential correlate and its $\log$ transformation within the binary analysis, and if significant, tertiles of the predictor were calculated. To consider the possible clustering effect within countries, multilevel analysis with a random intercept was performed with Stata 12 (StataCorp, College Station, TX, USA). Multilevel analysis was carried out for the final model (step three), including a null model (random-intercept-only) and a random intercept model.

\section{Results}

\section{Sample characteristics}

Two hundred women $(27.2 \%, 95 \%$ CI $[24.0,30.4])$ had a "low wellbeing" and 535 women (72.8\%) had a "high wellbeing" based on the pre-defined WHO-5 scores. In total, 561 women $(76.5 \%)$ were working and 690 (94.0\%) had a partner. $412(56.1 \%)$ women were highly educated (diploma or university), whereas 89 (12.1\%) had only a low education (no qualification or intermediate). Sample characteristics are displayed in Table 1 and Table 2 in detail.

Comparison of missing and non-missing data of resting heart rate showed no significant differences $(p \geq .05)$ in age, education, ethnicity, household composition, shift work, marital status, BMI, gestational week, pregnancyrelated worries, sleep hours, systolic and diastolic blood pressure. Nonetheless, significant differences were found between missing/non-missing data of resting heart rate and maternal mental health $(p<.01)$ and occupational status $(p<.05)$.

\section{Bivariate analysis}

Bivariate logistic regression models (first step) showed significant associations $(p<.10$, data not shown) between low wellbeing and age, BMI at baseline, weeks of gestation, ethnicity, household composition, education, occupational status, attitude to current weight, self-efficacy, social support, pregnancy-related worries (sociomedical, socioeconomic, health, relationship), marital status, pregnant before, shift work, hours of sleep per day, days of insufficient sleep per month, snoring, and systolic blood pressure.
No significant associations ( $p \geq .10$, data not shown) were displayed between low wellbeing and smoking, alcohol consumption, outcome expectancies, number of own children, previous stillbirths/miscarriages, previous fetal macrosomia, previous congenital malformation, PA, previous gestational diabetes, chronic hypertension, polycystic ovary syndrome, pre to baseline weight gain, fasting plasma glucose, fasting plasma insulin, HOMAIR, diastolic blood pressure, and resting heart rate.

\section{Multivariate analysis}

In a second step, four multivariate models (a-d) were developed. Remaining variables associated with socioeconomic status (ethnicity, household composition, education, occupational status, marital status, shift work) were analyzed in a first model (a). This model, $x^{2}(8, N$ $=727)=29.79, p<.01, R^{2}=.04$, showed significant associations between low wellbeing and ethnicity (European vs. not European: $O R=.52,95 \%$ CI $[.33, .83], p<.01)$, occupational status (not working vs. working: $O R=1.51$, 95\% CI [.94, 2.41], $p=.09$ ), marital status (with vs. without partner: $O R=.39,95 \% \mathrm{CI}[.18, .88], p=.02)$ and shift work (yes vs. no: $O R=1.79,95 \% \mathrm{CI}[1.19,2.69], p<.01$ ), but no longer with household composition $(p=.53)$ and education (low: $p=.61$, medium: $p=.13$ ).

The second model (b), $\chi^{2}(5, N=704)=53.44, p<.01$, $R^{2}=.07$, consisting of remaining variables related to sleeping quality (hours of sleep, days of insufficient sleep, snoring), showed significant associations of low wellbeing with hours of sleep per day ( 9 or more vs. normal: $O R=1.60,95 \%$ CI $[1.05,2.45], p=.03)$ and days of insufficient sleep per month (moderate vs. low: $O R=$ $1.73,95 \%$ CI $[1.08,2.77], p=.02$; high vs. low: $O R=4.13$, $95 \%$ CI $[2.63,6.48], p<.01)$, but no longer with snoring $(p=.32)$.

The third model (c), $\chi^{2}(4, N=720)=72.94, p<.01, R^{2}$ $=.10$, referring to remaining variables of the Cambridge Worry Scale, showed significant associations of low wellbeing with socioeconomic worries $(O R=1.11,95 \% \mathrm{CI}$ $[1.05,1.17], p<.01)$, health-related worries $(O R=1.06$, 95\% CI $[1.02,1.11], p<.01)$ and relationship-related worries $(O R=1.24,95 \%$ CI [1.12, 1.37], $p<.01)$, but no longer with sociomedical worries $(p=.90)$.

The fourth model (d), $\chi^{2}(3, N=725)=66.49, p<.01$, $R^{2}=.09$, referring to variables from the HAPA model, showed significant associations of low wellbeing and attitude to current weight $(O R=1.10,95 \%$ CI $[1.05,1.16]$, $p<.01)$, self-efficacy $(O R=.96,95 \%$ CI $[.93, .98], p<.01)$ as well as social support $(O R=.91,95 \%$ CI $[.87, .95]$, $p<.01)$.

In the third and final step, all remaining variables of step two were analyzed within one multivariate logistic regression model. Results are displayed in Table 3, showing that low wellbeing was significantly associated with 
Table 2 Specific maternal characteristics

\begin{tabular}{|c|c|c|c|}
\hline Characteristic & $\begin{array}{l}\text { Total } \\
(N=735)\end{array}$ & $\begin{array}{l}\text { Low wellbeing }^{\text {a }} \\
(n=200)\end{array}$ & $\begin{array}{l}\text { High wellbeing } \\
(n=535)\end{array}$ \\
\hline \multicolumn{4}{|l|}{ Lifestyle } \\
\hline Smoking behaviour, yes (n (\%)) & $122(16.6)$ & $37(18.5)$ & $85(15.9)$ \\
\hline Any alcohol consumption, yes $(n(\%))$ & $43(5.9)$ & $12(6.0)$ & $31(5.8)$ \\
\hline Sleep, hours per day (median (IQR), total = 734) & $8.0(7.0-8.5)$ & $8.0(7.0-9.0)$ & $8.0(7.0-8.0)$ \\
\hline $\begin{array}{l}\text { Insufficient sleep, days per month } \\
\text { (median (IQR, total = 722) }\end{array}$ & $7.0(3.0-15.0)$ & $15.0(5.0-20.0)$ & $5.0(2.0-15.0)$ \\
\hline Snoring, days per week (median (IQR, total = 714) & $0.0(0-2.0)$ & $0.0(0-3.0)$ & $0.0(0-2.0)$ \\
\hline \multicolumn{4}{|l|}{ PPAQ, MET-h.wk. ${ }^{-1}$ (median (IQR)) } \\
\hline MVPA & $70.2(34.7-127.8)$ & $74.7(32.3-128.2)$ & $69.0(35.5-127.8)$ \\
\hline SB & $42.0(19.1-62.1)$ & $36.8(18.6-61.1)$ & $43.2(19.4-62.7)$ \\
\hline \multicolumn{4}{|l|}{ Biological } \\
\hline First degree relative with diabetes, yes $(n(\%))$ & $168(22.9)$ & $48(24.0)$ & $120(22.4)$ \\
\hline Chronic hypertension, yes $(n(\%)$, total $=730)$ & $98(13.3)$ & $30(15.0)$ & $68(12.7)$ \\
\hline Polycystic ovary syndrome, yes $(n(\%)$, total $=728)$ & $73(9.9)$ & $24(12.0)$ & $49(9.2)$ \\
\hline Fasting plasma glucose, $\mathrm{mmol} / \mathrm{L}$ (median $(\mathrm{IQR})$, total = 727) & $4.6(4.3-4.8)$ & $4.6(4.3-4.8)$ & $4.6(4.3-4.8)$ \\
\hline Fasting plasma insulin, $\mathrm{mmol} / \mathrm{L}$ (median $(\mathrm{IQR})$, total $=720$ ) & $12.7(9.8-17.5)$ & $13.1(10.1-17.5)$ & $12.5(9.5-17.4)$ \\
\hline HOMA-IR (median (IQR), total = 717) & $2.6(1.9-3.6)$ & $2.7(2.0-3.6)$ & $2.6(1.9-3.6)$ \\
\hline Systolic blood pressure (median (IQR), total = 733) & $116(109-123)$ & $114(107-121)$ & $116(110-124)$ \\
\hline Diastolic blood pressure (median (IQR), total = 733) & $72(67-79)$ & $71(67-77)$ & $72(67-79)$ \\
\hline Resting heart rate (median (IQR), total=694) & $79(72-86)$ & $79(71-87)$ & $79(73-85)$ \\
\hline \multicolumn{4}{|l|}{ Pregnancy-specific } \\
\hline Gestational week (median (IQR)) & $15.1(13.4-16.7)$ & $14.9(13.3-16.2)$ & $15.3(13.6-17.0)$ \\
\hline \multicolumn{4}{|l|}{ Number of own children $(n(\%)$, total = 734) } \\
\hline Zero & $366(49.8)$ & $93(46.5)$ & $273(51.0)$ \\
\hline One & $256(34.8)$ & $73(36.5)$ & $183(34.2)$ \\
\hline Two or more & $112(15.2)$ & $34(17.0)$ & $78(14.6)$ \\
\hline Pregnant before, yes $(n(\%))$ & $460(62.6)$ & $136(68.0)$ & $324(60.6)$ \\
\hline Previous stillbirths/miscarriage, $\geq 1(n(\%)$, total $=445)$ & $200(27.2)$ & $63(31.5)$ & $137(25.6)$ \\
\hline Previous fetal macrosomia, yes $(n(\%)$, total $=453)$ & $81(11.0)$ & $22(11.0)$ & $59(11.0)$ \\
\hline Previous congenital malformation, yes $(n(\%)$, total $=454)$ & $16(2.2)$ & $4(2.0)$ & $12(2.2)$ \\
\hline Previous gestational diabetes, yes ( $n(\%)$, total $=455$ ) & $36(4.9)$ & $12(6.0)$ & $24(4.5)$ \\
\hline Pre to baseline weight gain, $\mathrm{kg}$ (median (IQR)) & $1.7(-0.4-4.0)$ & $1.6(-0.6-4.2)$ & $1.7(-0.3-4.0)$ \\
\hline \multicolumn{4}{|l|}{ Perceptions and attitude ${ }^{\mathrm{b}}$ (median (IQR)) } \\
\hline Attitude to current weight (total = 729) & $16.0(14.0-18.0)$ & $17.0(14.0-19.0)$ & $16.0(13.0-18.0)$ \\
\hline Self-efficacy (total = 730) & $35.0(30.0-41.0)$ & $31.0(27.0-38.0)$ & $37.0(31.0-41.0)$ \\
\hline Social support (total = 731) & $16.0(13.0-18.0)$ & $14.0(10.0-17.0)$ & $16.0(14.0-18.0)$ \\
\hline Outcome expectancy (total = 723) & $52.0(46.0-58.0)$ & $52.0(46.0-58.0)$ & $52.0(46.0-58.0)$ \\
\hline \multicolumn{4}{|l|}{ Cambridge worry scale (median (IQR)) } \\
\hline Total 13 items (total = 720) & $18.0(12.0-26.0)$ & $24.0(16.0-32.0)$ & $17.0(12.0-24.0)$ \\
\hline Sociomedical (total = 731) & $5.0(2.0-8.0)$ & $6.0(2.0-10.0)$ & $5.0(2.0-8.0)$ \\
\hline Socioeconomic (total = 733) & $4.0(2.0-7.0)$ & $6.0(3.0-9.0)$ & $4.0(1.0-6.0)$ \\
\hline Health (total $=729$ ) & $8.0(5.0-12.0)$ & $10.0(6.0-13.0)$ & $8.0(5.0-11.0)$ \\
\hline Relationship (total = 729) & $0.0(0-2.0)$ & $1.0(0-3.0)$ & $0.0(0-1.0)$ \\
\hline
\end{tabular}

$\mathrm{PPAQ}=$ pregnancy physical activity questionnaire; $\mathrm{MET}=$ metabolic equivalents; $\mathrm{MVPA}=$ moderate-to-vigorous physical activity; $\mathrm{SB}=$ sedentary behavior a Based on the World Health Organization Well-Being Index (WHO-5). Values $\leq 50$ were considered as low wellbeing (poor maternal mental health), values $>50$ as high wellbeing. ${ }^{\text {b }}$ ased on the Health Action Process Approach (HAPA) model [43] 
Table 3 Multivariate logistic regression. Associations of low wellbeing and maternal characteristics

\begin{tabular}{|c|c|c|c|c|}
\hline \multirow[b]{2}{*}{ Maternal characteristic } & \multicolumn{3}{|c|}{ Odds Ratio, 95\% Cl } & \multirow[b]{2}{*}{$p$} \\
\hline & $O R$ & Lower & Upper & \\
\hline Ethnicity (European) & .44 & .25 & .77 & $<.01^{*}$ \\
\hline Marital status (with partner) & .45 & .20 & 1.02 & .06 \\
\hline Shift work (yes) & 1.81 & 1.11 & 2.93 & $.02^{*}$ \\
\hline \multicolumn{5}{|c|}{ Occupational status (working = ref) } \\
\hline Not working & 1.39 & .80 & 2.42 & .24 \\
\hline Home duties & 1.28 & .59 & 2.78 & .53 \\
\hline \multicolumn{5}{|l|}{ Sleep $h / d(6-9 h=r e f)^{a}$} \\
\hline$\leq 6 \mathrm{~h}$ & .90 & .52 & 1.56 & .70 \\
\hline$\geq 9 h$ & 1.11 & .68 & 1.84 & .67 \\
\hline \multicolumn{5}{|c|}{ Insufficient sleep $\mathrm{d} / \mathrm{m}(\mathrm{low}=\mathrm{ref})^{\mathrm{b}}$} \\
\hline Medium & 1.26 & .73 & 2.17 & .41 \\
\hline High & 3.30 & 1.96 & 5.55 & $<.01^{*}$ \\
\hline \multicolumn{5}{|l|}{ Perceptions and attitude ${ }^{c}$} \\
\hline Attitude to current weight & 1.05 & .99 & 1.12 & .11 \\
\hline Self-efficacy & .95 & .92 & .98 & $<.01^{*}$ \\
\hline Social support & .94 & .90 & .99 & $.03^{*}$ \\
\hline \multicolumn{5}{|l|}{ Cambridge worry scale } \\
\hline Socioeconomic & 1.08 & 1.02 & 1.15 & $.01^{*}$ \\
\hline Health & 1.06 & 1.01 & 1.11 & $.03^{*}$ \\
\hline Relationship & 1.17 & 1.05 & 1.31 & $<.01^{*}$ \\
\hline \multicolumn{5}{|l|}{ Age $(\text { young }=\text { ref })^{d}$} \\
\hline middle & .81 & .50 & 1.31 & .39 \\
\hline old & .90 & .54 & 1.49 & .68 \\
\hline BMI at baseline & 1.03 & .98 & 1.08 & .32 \\
\hline Weeks of gestation & .95 & .87 & 1.03 & .17 \\
\hline Pregnant before (yes) & 1.11 & .72 & 1.71 & .64 \\
\hline Systolic blood pressure & .99 & .97 & 1.01 & .21 \\
\hline
\end{tabular}

Only variables remaining after step two were included in the model (method $=$ enter, outcome $=$ WHO-5 index: low wellbeing), $\chi 2(21, N=692)=163.71, p$ $<.001, R^{2}=.21$, Durbin Watson $=2.10$

${ }^{a}$ sleep hours per day ( $24 \mathrm{~h}$, self-reported); $\leq 6 \mathrm{~h} ; 6-9 \mathrm{~h} ; \geq 9 \mathrm{~h}$

${ }^{b}$ days per month (self-reported), based on tertiles

'Based on the Health Action Process Approach (HAPA) model [43]

dbased on tertiles

$*$ * 05

non-European ethnicity, shift work, insufficient sleep, low self-efficacy, low social support and pregnancyrelated worries (socioeconomic, health, relationship), $x^{2}$ $(21, N=692)=163.71, p<.001, R^{2}=.21$.

Although multilevel analysis revealed significant level 2 variances for country, $\tau_{00}=.10,95 \%$ CI $[.02, .48], \rho$ $=.03, p<.05$, in the null model (random-intercept-only), there was no significant improvement of the final model in step 3, when adding a random intercept. Therefore, results of step 3 are presented without a random intercept (Table 3).

\section{Discussion}

In summary, this study showed independent associations between low wellbeing and non-European ethnicity, shift work, insufficient sleep, low self-efficacy, low social support and pregnancy-related worries (socioeconomic, health, relationship) in obese pregnant women.

A recent meta-analysis [15] on the prevalence of depressive symptoms in pregnancy showed higher rates for obese (33\%) than normal-weight pregnant women (22.6\%). Likewise, in the present study, $27.2 \%$ of all women with $\mathrm{BMI} \geq$ 29 had a low wellbeing. There is a reciprocal relationship between poor mental health and obesity [11] and both have been linked with numerous adverse pregnancy outcomes such as neonatal intensive care requirement, preterm birth and gestational diabetes [24, 32, 33]. Thus, healthcare providers should be aware of the high prevalence of obese pregnant women experiencing mental health problems.

A number of studies reported a broad range of potential correlates of maternal mental health [16, 17], although some variability appears, largely based either on bivariate or multivariate analysis, sample composition and study design. For example, the present study demonstrated an association between maternal mental health and European ethnicity, which is in line with previous studies, showing both independent and mutual influences of ethnicity and variables of socioeconomic status (e.g., occupation, education, income) on maternal mental health $[16,44,45]$. Likewise, sleep quality is associated with depression in the general population [46] and during pregnancy, where poor sleep quality and sleep loss are linked with a greater risk of both maternal mood problems [47] and adverse birth outcomes [48]. This confirms our finding of the linkage between low wellbeing and insufficient sleep. In addition, previous studies confirm our findings of associations between low wellbeing and individual variables such as low self-efficacy, worrying and less perceived social support as well [16].

In contrast to other studies, we did not find associations (neither univariate nor multivariate) for previously described correlates of mental health in pregnancy such as age, alcohol consumption, smoking, number of own children or previous pregnancy complications [16, 17]. This may be due to the selection of women participating in the DALI study, small frequencies for some correlates (e.g., alcohol consumption, previous congenital malformation, previous gestational diabetes) or restriction of inclusion for BMI and gestational week.

Interestingly, no association between low wellbeing and PA was found, in contrast to previous research $[18,49,50]$. This may be related to the study context, where women were included in a lifestyle trial, partly focused on physically activity. Moreover, we studied women in early pregnancy, while the decrease in PA may be more pronounced later in pregnancy [18]. 
According to pregnancy-related worries, pregnancy is a sensitive period in a woman's life, and is likely to evoke heightened levels of anxiety and worrying [51] with unique influences on mood outcomes [42]. As shown in the present study, pregnancy-related worries should be considered as possible correlates of poor maternal mental health states. Furthermore, it may be possible that pregnancy-related worries play a crucial role in intervention strategies on improving maternal mental health. However, in pursuance of achieving the best outcomes when developing such intervention strategies, more insight into the relevance of a woman's current worries in pregnancy and how to deal with them is needed.

Under the terms of the health action process approach [43], social support and self-efficacy are important factors for forming intentions and maintaining changes in health behaviors (e.g., for PA or HE). Since many studies reported an inverse relationship between depression in pregnancy and PA [18], it could be possible that lack of social support and low self-efficacy deteriorate the unfavorable effects of an unhealthy lifestyle on mental and physical health. These effects might be even stronger in late pregnancy. Considering low social support and selfefficacy as correlates might help for an early recognition of poor maternal mental health states and the design of interventions. In particular, implementing intervention strategies, which include for instance, participation of family members and friends in order to increase social support or specific tasks to increase perceived selfefficacy are advisable. The inclusion of such individual characteristics into tailored intervention strategies would probably result in the best outcomes of maternal mental health.

Finally, the well-known association between poor mental health and shift work [52] was confirmed in pregnancy as well. In short, working in shifts (e.g., night work, rotating shifts) disturbs the circadian rhythm, a dynamic system which regulates most of our physiological processes based on the day-night cycle, and which is associated with pregnancy complications such as pre-term birth or low birth weight [53]. Together with sleep deprivation, the disruption of the circadian system could act as stressor with detrimental consequences on many body systems, including woman's reproductive function, while leading to a cumulative wear out of the whole system [54]. With this in view, integrating shift work as a correlate of poor maternal mental health is highly recommended.

During the last decade, researchers have shown an increased interest in mental health during pregnancy. However, the use of maternal mental health correlates for early recognition of poor mental health into clinical practice seems to be limited as yet. Clearly, some of the identified correlates would be difficult to routinely assess in clinical practice but others (e.g., non-European ethnicity, worrying, insufficient sleep, shift work) could be easily assessed without the requirement of laboratory equipment or standardized questionnaires. Such an implementation (e.g., for screening) would be possible both in primary (e.g., general practitioners, midwifes) and secondary health care settings (obstetricians) and could help in early recognition of maternal mental health problems at a low threshold. In addition, identified correlates give direction to further intervention development for prevention or treatment of mental health problems in pregnancy. Finally, because of the reciprocal relationship between obesity and poor mental health, tackling mental health problems of obese pregnant women might help to mitigate part of the adverse effects of obesity on pregnancy outcomes.

\section{Limitations and strengths}

Our study has limitations and strengths that deserve to be mentioned. First, due to the cross-sectional design, no conclusions about causality can be drawn. To confirm whether the identified factors are indeed predictors for low mood in the course of pregnancy and to establish the consequences for both the woman and the child, longitudinal studies are warranted. In addition, the cross-sectional design and the chosen analysis strategy hinders the detection of causal pathways of variables associated with mental health in pregnancy. However, this study focused on the detection of correlates of poor mental health rather than causal pathways. Secondly, our data were derived from a large group of women from different European countries participating in the DALI study who might have a better mental health compared to others, possibly leading to a selection bias.

Yet, 27\% reported low wellbeing, as defined by their WHO-5 score, which is in concert with previous research on other populations, and thus does not suggest an underestimation. Of course, we need to acknowledge that we used a relatively short measure of mental health (subjective wellbeing) rather than a diagnostic interview, which is the gold standard for establishing the true prevalence of major depression. Such a procedure was not feasible in the context of this large multi-national lifestyle study. Regardless, the WHO-5 scale has shown good screening properties for clinically relevant depressive symptoms, was used worldwide and tested in all languages required in the present study, as well as variations of the scale dependent on country of recruitment were statistically evaluated in the present study $[38,39]$. Therefore, a bias is very unlikely and our data can be regarded as valid. Future studies should aim to thoroughly investigate the prevalence and course of minor and major depression in pregnancy. 
Nonetheless, to our knowledge, this is the first study, which investigated a broad range of potential correlates of poor maternal mental health in a crucial period such as the early pregnancy in conjunction with a wide and representative European sample.

\section{Conclusions}

One of the great challenges is to recognize maternal mental health problems very early in pregnancy in order to prevent harmful health consequences. This study showed that mental health problems are common in European obese pregnant women (27.2\%) and that nonEuropean ethnicity, having shift work, insufficient sleep, low self-efficacy, low social support, and pregnancyrelated worries have to be considered as correlates of poor maternal mental health in this population. Other factors such as smoking, alcohol consumption, marital status, occupational status and maternal age do not seem be associated with maternal mental health in this population. Some of these identified correlates may further help for an early recognition in clinical practice and the design of interventions. Especially insufficient sleep and non-European ethnicity seem to play a prominent role according to their effect sizes.

\section{Abbreviations}

BMI: body mass index; HAPA: health action process approach; HE: healthy eating; HOMA-IR: homeostasis model assessment of insulin resistance; IQR: interquartile range; MET: metabolic equivalent of task; MVPA: moderateto-vigorous physical activity; PA: physical activity; PPAQ: pregnancy physical activity questionnaire; SB: sedentary behavior; WHO: world health organization

\section{Acknowledgments}

The authors thank Kenneth B. Smale for proofreading the manuscript.

\section{Funding}

The DALI project was registered as an RCT (ISRCTN70595832) and received funding from the European Community's 7th Framework Programme (FP7/ 2007-2013) under grant agreement no 242187. In the Netherlands, further funding was provided by the Netherlands Organisation for Health Research and Development (ZonMw; grant number: 200,310,013). The funders had no role in any aspect of the study beyond funding.

\section{Availability of data and materials}

The dataset used and/or analyzed during this current study is available from the corresponding author on reasonable request.

\section{Authors' contributions}

MS contributed to the data analysis and writing of the manuscript and took the lead in redrafting the script following editorial review. DS coordinated the study. DS GD RC JA AKW FAA RD DH PD EM EWO AZ AL MD AB FD DJ LLA FS MP contributed to the conception and design of the study. KB GJ JJ SG JH contributed to the acquisition of data. MS AB MP contributed to the analysis and interpretation of data. All authors read and corrected draft versions of the manuscript and approved the final manuscript.

\section{Ethics approval and consent to participate}

All procedures performed in studies involving human participants were in accordance with the ethical standards of the institutional and/or national research committee and with the 1964 Helsinki declaration and its later amendments or comparable ethical standards. For each country, the study design and procedures were approved by the local Medical Ethics committee (Amsterdam, the Netherlands: Ethical Committee of the VU
University Medical Center; Barcelona, Spain: Comité Ético de Investigación Clínica de la Fundació de Gestió Sanitaria del Hospital de la Santa Creu i Sant Pau; Cambridge, United Kingdom: National Research Ethics Service, Norwich Research Ethics Committee; Copenhagen and Odense, Denmark: De Videnskabsetiske Komiteer D for Region Hovedstaden; Galway, Ireland: Clinical Research Ethics Committee, Galway University Hospitals; Leuven, Belgium: Commissie Medische Ethiek van de Universitaire Ziekenhuizen KU Leuven; Padua, Italy: II Comitato Etico per la Sperimentazione Clinica della Provincia di Padova; Poznan, Poland: Komisja Bioetyczna Przy Uniwersytecie Medycznym im. Karola Marcinkowskiego W Poznaniu; Vienna, Austria: Ethik Kommission Medizinische Universität Wien). Written informed consent was obtained from all individual participants included in the study. Trial registration details: ISRCTN70595832, 02.12.2011.

\section{Consent for publication \\ Not applicable.}

\section{Competing interests}

The authors declare that they have no competing interests.

\section{Publisher's Note}

Springer Nature remains neutral with regard to jurisdictional claims in published maps and institutional affiliations.

\section{Author details}

${ }^{1}$ Institute of Sport Science, University of Graz, Mozartgasse 14, 8010, Graz, Austria. ${ }^{2}$ Department of Public and Occupational Health, Amsterdam Public Health Research Institute, VU University Medical Centre, Amsterdam, the Netherlands. ${ }^{3}$ Department of Development and Regeneration KULeuven, University of Leuven, Leuven, Belgium and Faculty of Medicine and Health Sciences, Centre for Research and Innovation in Care (CRIC), University of Antwerp, Belgium and Faculty of Health and Social Work, research unit Healthy Living, UC Leuven-Limburg, Leuven, Belgium. ${ }^{4}$ Institute of Metabolic Science, Addenbrookes Hospital, Cambridge, England and Macarthur Clinical School, Western Sydney University, Sydney, Australia. ${ }^{5}$ Department of Obstetrics and Gynecology, Medizinische Universität Graz, Graz, Austria. ${ }^{6}$ Institut de Recerca de L'Hospital de la Santa Creu i Sant Pau, Barcelona, Spain. ${ }^{7}$ CIBER Bioengineering, Biomaterials and Nanotechnology, Instituto de Salud Carlos III, Zaragaza, Spain. ${ }^{8}$ Division of Endocrinology and Metabolism, Department of Medicine III, Medical University of Vienna, Vienna, Austria. ${ }^{9} \mathrm{KU}$ Leuven Department of Development and Regeneration: Pregnancy, Fetus and Neonate, Gynaecology and Obstetrics, University Hospitals, Leuven, Belgium. ${ }^{10}$ Department of Obstetrics and Gynaecology Division of Obstetrics and Prenatal Medicine, Erasmus Medical Centre, Rotterdam, The Netherlands.

${ }^{11}$ Recherche en Santé Lawson SA, Bronschhofen, Switzerland. ${ }^{12}$ Center for Pregnant Women with Diabetes, Departments of Endocrinology and Obstetrics, Rigshospitalet, University of Copenhagen, Copenhagen, Denmark. ${ }^{13}$ Division of Reproduction, Poznan University of Medical Sciences, Poznan, Poland. ${ }^{14}$ Universita Degli Studi di Padova, Padova, Italy. ${ }^{15}$ Università di Pisa, Pisa, Italy. ${ }^{16}$ National University of Ireland, Galway, Ireland. ${ }^{17}$ Odense University Hospital, Odense, Denmark. ${ }^{18}$ Department of Medical Psychology, VU University Medical Centre, Amsterdam, the Netherlands. ${ }^{19}$ Department of Medical Psychology, Academic Medical Centre, Amsterdam, the Netherlands.

Received: 13 April 2017 Accepted: 24 November 2017

Published online: 04 December 2017

\section{References}

1. World Health Organization Mental health-facing the challenges, building solutions: report from the WHO European ministerial conference: World $\mathrm{H}$ ealth Organization; 2005.

2. Steel Z, Marnane C, Iranpour C, Chey T, Jackson JW, Patel V, Silove D. The global prevalence of common mental disorders: a systematic review and meta-analysis 1980-2013. Int J Epidemiol. 2014;43:476-93. doi:10.1093/ije/ dyu038.

3. Kessler RC, Bromet EJ. The epidemiology of depression across cultures. Annu Rev Public Health. 2013;34:119-38. doi:10.1146/annurev-publhealth031912-114409.

4. Prince M, Patel V, Saxena S, Maj M, Maselko J, Phillips MR, Rahman A. No health without mental health. Lancet. 2007:370:859-77. doi:10.1016/S01406736(07)61238-0 
5. Russo J, Katon W, Sullivan M, Clark M, Buchwald D. Severity of somatization and its relationship to psychiatric disorders and personality. Psychosomatics. 1994;35:546-56.

6. Compare A, Zarbo C, Manzoni GM, Castelnuovo G, Baldassari E, Bonardi A et al. Social support, depression, and heart disease: a ten year literature review. Front Psychol. 2013; doi:10.3389/fpsyg.2013.00384 .

7. Cohen BE, Edmondson D, Kronish IM. State of the art review: depression, stress, anxiety, and cardiovascular disease. Am J Hypertens. 2015;28:1295302. doi:10.1093/ajh/hpv047

8. Holt RIG, de Groot M, Golden SH. Diabetes and depression. Curr Diab Rep. 2014; doi: 10.1007/s11892-014-0491-3.

9. Ciesla JA, Roberts JE. Meta-analysis of the relationship between HIV infection and risk for depressive disorders. AJP. 2001;158:725-30. doi:10. 1176/appi.ajp.158.5.725.

10. Sweetland A, Oquendo M, Wickramaratne P, Weissman M, Wainberg M. Depression: a silent driver of the global tuberculosis epidemic. World Psychiatry. 2014;13:325-6. doi:10.1002/wps.20134.

11. Luppino FS, de Wit LM, Bouvy PF, Stijnen T, Cuijpers P, Penninx BWJH, Zitman FG. Overweight, obesity, and depression. Arch Gen Psychiatry. 2010; 67:220. doi:10.1001/archgenpsychiatry.2010.2.

12. Kivimaki M, Batty GD, Singh-Manoux A, Nabi H, Sabia S, Tabak AG, et al. Association between common mental disorder and obesity over the adult life course. Br J Psychiatry. 2009;195:149-55. doi:10.1192/bjp.bp. 108.057299

13. Gavin NI, Gaynes BN, Lohr KN, Meltzer-Brody S, Gartlehner G, Swinson T. Perinatal depression: a systematic review of prevalence and incidence. Obstet Gynecol. 2005;106:1071-83.

14. Howard LM, Molyneaux E, Dennis C-L, Rochat T, Stein A, Milgrom J. Nonpsychotic mental disorders in the perinatal period. Lancet. 2014;384:177588. doi:10.1016/S0140-6736(14)61276-9

15. Molyneaux E, Poston L, Ashurst-Williams S, Howard LM. Obesity and mental disorders during pregnancy and postpartum: a systematic review and metaanalysis. Obstet Gynecol. 2014;123:857-67. doi:10.1097/AOG. 0000000000000170

16. Biaggi A, Conroy S, Pawlby S, Pariante CM. Identifying the women at risk of antenatal anxiety and depression: a systematic review. J Affect Disord. 2016: 191:62-77. doi:10.1016/j.jad.2015.11.014

17. Lancaster CA, Gold KJ, Flynn HA, Yoo H, Marcus SM, Davis MM. Risk factors for depressive symptoms during pregnancy: a systematic review. Am J Obstet Gynecol. 2010;202:5-14. doi:10.1016/j.ajog.2009.09.007

18. Poudevigne MS, O'Connor PJA. Review of physical activity patterns in pregnant women and their relationship to psychological health. Sports Med. 2006;36:19-38.

19. Daley AJ, Foster L, Long G, Palmer C, Robinson O, Walmsley H, Ward R. The effectiveness of exercise for the prevention and treatment of antenatal depression: systematic review with meta-analysis. BJOG. 2015;122:57-62. doi: 10.1111/1471-0528.12909

20. Downs DS, Chasan-Taber L, Evenson KR, Leiferman J, Yeo S. Physical activity and pregnancy. Res Q Exerc Sport. 2012;83:485-502. doi:10.1080/02701367. 2012.10599138

21. Barker DJP. The origins of the developmental origins theory. J Intern Med. 2007;261:412-7. doi:10.1111/j.1365-2796.2007.01809.x

22. Bale TL, Baram TZ, Brown AS, Goldstein JM, Insel TR, McCarthy MM, et al. Early life programming and neurodevelopmental disorders. Biol Psychiatry. 2010;68:314-9. doi:10.1016/j.biopsych.2010.05.028

23. Kim DR, Bale TL, Epperson CN. Prenatal programming of mental illness: current understanding of relationship and mechanisms. Curr Psychiatry Rep. 2015; doi:10.1007/s11920-014-0546-9

24. Stein A, Pearson RM, Goodman SH, Rapa E, Rahman A, McCallum M, et al. Effects of perinatal mental disorders on the fetus and child. Lancet. 2014; 384:1800-19. doi:10.1016/S0140-6736(14)61277-0 .

25. Accortt EE, Cheadle ACD, Dunkel Schetter C. Prenatal depression and adverse birth outcomes: an updated systematic review. Matern Child Health J. 2015:19:1306-37. doi:10.1007/s10995-014-1637-2

26. Szegda K, Markenson G, Bertone-Johnson ER, Chasan-Taber L. Depression during pregnancy: a risk factor for adverse neonatal outcomes? A critical review of the literature. J Matern Fetal Neonatal Med. 2013;27:960-7. doi:10. 3109/14767058.2013.845157

27. O'Connor TG, Monk C, Fitelson EM. Practitioner review: maternal mood in pregnancy and child development-implications for child psychology and psychiatry. J Child Psychol Psychiatry. 2014;55:99-111. doi:10.1111/jcpp.12153.
28. Kingston D, McDonald S, Austin M-P, Tough S. Association between prenatal and postnatal psychological distress and toddler cognitive development: a systematic review. PLoS One. 2015;10:e0126929. doi:10. 1371/journal.pone.0126929.

29. Howard LM, Piot P, Stein A. No health without perinatal mental health. Lancet. 2014;384:1723-4. doi:10.1016/S0140-6736(14)62040-7 .

30. Fisher SC, Kim SY, Sharma AJ, Rochat R, Morrow B. Is obesity still increasing among pregnant women? Prepregnancy obesity trends in 20 states, 20032009. Prev Med. 2013;56:372-8. doi:10.1016/j.ypmed.2013.02.015 .

31. Heslehurst N, Ells L, Simpson H, Batterham A, Wilkinson J, Summerbell CD. Trends in maternal obesity incidence rates, demographic predictors, and health inequalities in 36,821 women over a 15-year period. BJOG. 2007;114: 187-94. doi:10.1111/j.1471-0528.2006.01199.x.

32. Heslehurst N, Simpson H, Ells LJ, Rankin J, Wilkinson J, Lang R, et al. The impact of maternal BMI status on pregnancy outcomes with immediate short-term obstetric resource implications: a meta-analysis. Obes Rev. 2008; 9:635-83. doi:10.1111/j.1467-789X.2008.00511.x.

33. O'Reilly JR, Reynolds RM. The risk of maternal obesity to the long-term health of the offspring. Clin Endocrinol. 2013;78:9-16. doi:10.1111/cen.12055

34. Simmons D, Jelsma JGM, Galjaard S, Devlieger $R$, van Assche A, Jans $G$, et al. Results from a European multicenter randomized trial of physical activity and/or healthy eating to reduce the risk of gestational diabetes mellitus: the DALI lifestyle pilot. Diabetes Care. 2015;38:1650-6. doi:10.2337/dc15-0360

35. Jelsma JGM, van Poppel MNM, Galjaard S, Desoye G, Corcoy R, Devlieger R, et al. DALI: vitamin D and lifestyle intervention for gestational diabetes mellitus (GDM) prevention: an European multicentre, randomised trial study protocol. BMC Pregnancy Childbirth. 2013;13:142. doi:10.1186/14712393-13-142

36. Vellinga A, Zawiejska A, Harreiter J, Buckley B, Di Cianni G, Lapolla A, et al. Associations of body mass index (maternal BMI) and gestational diabetes mellitus with neonatal and maternal pregnancy outcomes in a multicentre European database (diabetes and pregnancy vitamin D and lifestyle intervention for gestational diabetes mellitus prevention). ISRN Obes. 2012; 2012:424010. doi:10.5402/2012/424010 .

37. World Health Organization. Wellbeing measures in primary health care: the DepCare project: report on a WHO meeting Stockholm, Sweden 12-13 February 1998: WHO Regional Office for Europe; 1998.

38. Topp CW, Østergaard SD, Søndergaard S, Bech P. The WHO-5 well-being index: a systematic review of the literature. Psychother Psychosom. 2015;84: 167-76. doi:10.1159/000376585.

39. Krieger T, Zimmermann J, Huffziger S, Ubl B, Diener C, Kuehner C, Grosse Holtforth M. Measuring depression with a well-being index: further evidence for the validity of the WHO well-being index (WHO-5) as a measure of the severity of depression. J Affect Disord. 2014;156:240-4. doi: 10.1016/j.jad.2013.12.015

40. Grandner MA, Patel NP, Gehrman PR, Perlis ML, Pack Al. Problems associated with short sleep: bridging the gap between laboratory and epidemiological studies. Sleep Med Rev. 2010;14:239-47. doi:10.1016/j.smv.2009.08.001.

41. Chasan-Taber L, Schmidt MD, Roberts DE, Hosmer D, Markenson G, Freedson PS. Development and validation of a pregnancy physical activity questionnaire. Med Sci Sports Exerc. 2004;36:1750-60. doi:10.1249/01.MSS. 0000142303.49306.0D

42. Green JM, Kafetsios K, Statham HE, Snowdon CM. Factor structure, validity and reliability of the Cambridge worry scale in a pregnant population. J Health Psychol. 2003;8:753-64. doi:10.1177/13591053030086008 .

43. Schwarzer R. Modeling health behavior change: how to predict and modify the adoption and maintenance of health behaviors. Appl Psychol. 2008;57: 1-29. doi:10.1111/j.1464-0597.2007.00325.x.

44. Prady SL, Pickett KE, Croudace T, Fairley L, Bloor K, Gilbody S, et al. Psychological distress during pregnancy in a multi-ethnic community: findings from the born in Bradford cohort study. PLoS One. 2013;8:e60693. doi:10.1371/journal.pone.0060693

45. Fellenzer JL, Cibula DA. Intendedness of pregnancy and other predictive factors for symptoms of prenatal depression in a population-based study. Matern Child Health J. 2014:18:2426-36. doi:10.1007/s10995-014-1481-4 .

46. Murphy MJ, Peterson MJ. Sleep disturbances in depression. Sleep Medicine Clinics. 2015;10:17-23. doi:10.1016/j.jsmc.2014.11.009.

47. Bei B, Coo S, Trinder J. Sleep and mood during pregnancy and the postpartum period. Sleep Medicine Clinics. 2015;10:25-33. doi:10.1016/j.jsmc 2014.11.011. 
48. Palagini L, Gemignani A, Banti S, Manconi M, Mauri M, Riemann D. Chronic sleep loss during pregnancy as a determinant of stress: impact on pregnancy outcome. Sleep Med. 2014;15:853-9. doi:10.1016/j.sleep.2014.02.013 .

49. Gaston A, Prapavessis H. Tired, moody and pregnant? Exercise may be the answer. Psychol Health. 2013;28:1353-69. doi:10.1080/08870446.2013.809084

50. de Wit L, Jelsma JGM, van Poppel MNM, Bogaerts A, Simmons D, Desoye G, et al. Physical activity, depressed mood and pregnancy worries in European obese pregnant women: results from the DALI study. BMC Pregnancy Childbirth. 2015;15:158. doi:10.1186/s12884-015-0595-z.

51. Gourounti K, Anagnostopoulos F, Lykeridou K, Griva F, Vaslamatzis G. Prevalence of women's worries, anxiety, and depression during pregnancy in a public hospital setting in Greece. Clinical and experimental obstetrics \& gynecology. 2012;40:581-3.

52. Foster RG, Wulff K. The rhythm of rest and excess. Nat Rev Neurosci. 2005;6: 407-14. doi:10.1038/nrn1670.

53. Gamble KL, Resuehr D, Johnson CH. Shift work and circadian dysregulation of reproduction. Front Endocrinol (Lausanne). 2013;4:92. doi:10.3389/fendo. 2013.00092 .

54. McEwen BS, Karatsoreos IN. Sleep deprivation and circadian disruption: stress, Allostasis, and allostatic load. Sleep Medicine Clinics. 2015;10:1-10. doi:10.1016/j.jsmc.2014.11.007.

Submit your next manuscript to BioMed Central and we will help you at every step:

- We accept pre-submission inquiries

- Our selector tool helps you to find the most relevant journal

- We provide round the clock customer support

- Convenient online submission

- Thorough peer review

- Inclusion in PubMed and all major indexing services

- Maximum visibility for your research

Submit your manuscript at www.biomedcentral.com/submit
Biomed Central 